\title{
A NEW MULTI-CRITERIA EVALUATION MODEL BASED ON THE COMBINATION OF NON-ADDITIVE FUZZY AHP, CHOQUET INTEGRAL AND SUGENO $\lambda$-MEASURE
}

\author{
S. Nadi a*, M. Samiei ${ }^{\text {b }}$, H. R. Salari ${ }^{\text {b }}$, N. Karami ${ }^{b}$ \\ a Assistant Professor, Department of Geomatics Engineering, Faculty of Civil and Transportation Engineering, University of Isfahan, \\ Isfahan, Iran - snadi@eng.ui.ac.ir \\ ${ }^{\mathrm{b}}$ M.Sc. Remote sensing, Department of Geomatics Engineering, Faculty of Civil and Transportation Engineering, University of \\ Isfahan, Iran - mohammad.Samiei@eng.ui.ac.ir, h.salari@eng.ui.ac.ir, ni.karami@yahoo.com
}

KEY WORDS: Multi-criteria Evaluation, Uncertainty, Fuzzy AHP, Fuzzy Ranking, Choquet Integral, Sugeno $\lambda$-Measure

\begin{abstract}
:
This paper proposes a new model for multi-criteria evaluation under uncertain condition. In this model we consider the interaction between criteria as one of the most challenging issues especially in the presence of uncertainty. In this case usual pairwise comparisons and weighted sum cannot be used to calculate the importance of criteria and to aggregate them. Our model is based on the combination of non-additive fuzzy linguistic preference relation AHP (FLPRAHP), Choquet integral and Sugeno $\lambda$-measure. The proposed model capture fuzzy preferences of users and fuzzy values of criteria and uses Sugeno $\lambda$-measure to determine the importance of criteria and their interaction. Then, integrating Choquet integral and FLPRAHP, all the interaction between criteria are taken in to account with least number of comparison and the final score for each alternative is determined. So we would model a comprehensive set of interactions between criteria that can lead us to more reliable result. An illustrative example presents the effectiveness and capability of the proposed model to evaluate different alternatives in a multi-criteria decision problem.
\end{abstract}

\section{INTRODUCTION}

In most multi-criteria evaluation problems, the comprehension of the interaction between criteria is very informative especially under uncertain condition. The uncertainties can be raised from user's ambiguity about their preferences, imprecise criteria values and modeling process. By interaction we mean the existence of positive or negative synergy between criteria.

Recently, a number of researches have addressed the issue of interaction between criteria in multi-criteria evaluation problems (Greco et.al. 2014, Grabisch 1996, Kojadinovic 2002). considering the interaction between the criteria to be in the form of non-linear network structure, is the solution that is employed by ANP method (Saaty 1996 and 2005). Creating such a structure between the criteria is difficult even for experts and the sensitivity of the results to the structure of the network is a challenging issue. Furthermore, in order to model human decision-making process, it is better to use fuzzy measures which does not need the assumptions of additivity and independency among decision criteria. Sugeno (1974) introduced the concept of fuzzy $\lambda$-measure. Sugeno replaced the additively requirement of normal (classical) measures with weaker requirement of monotonicity and continuity. This concept used as a powerful tool to model the interaction phenomenon in decision-making (Grabisch 1995, Kojadinovic 2002). As an aggregation operator Choquet Integral proposed by many authors as a suitable alternative for weighted arithmetic mean or OWA operator to aggregate interaction between criteria (Grabisch 1995, Grabisch et.al. 2000, Labreuche and Grabisch and Grabisch 2003). In Choquet integral model, criteria can be interdependent and a fuzzy measure used to assign weights to each combination of criteria and make it possible to model the interaction between criteria. One of the Choquet integral drawbacks is lack of proper structure for the problem. Arranging component of the decision in a hierarchical structure, provides an overall view of the complex relationships between components. Extending AHP to include the interaction among criteria as well as different kinds of uncertainty in the evaluation process provides an interesting model. Such a model can be benefitted from the advantages of AHP such as its simple hierarchical structure, flexibility and the ability to model both qualitative and quantitative criteria and also provides the ability to model criteria which act conjunctively as well as criteria that act disjunctively under uncertainty (Grabisch et.al. 2000).

In this paper we proposed a new model to include the ability of modelling criteria interaction in the AHP under uncertain condition. The model is based on the combination of fuzzy linguistic preference relation AHP (FLPRAHP) method, Sugeno $\lambda$-measure and Choquet integral. FLPRAHP is used to organize the problem and the criteria and determine users' preferences and criteria values under uncertain condition. Choquet integral and Sugeno $\lambda$-measure aggregate users' preferences and criteria values to determine the overall score of each alternative by considering interaction among criteria (Sugeno 1974, Grabisch et.al. 2000, Labreuche and Grabisch 2003). The model uses fuzzy users' preferences and fuzzy criteria values. Then, Sugeno $\lambda$ measure method is used to determine the weights of importance for each criterion and any coalition of them. Afterwards Choquet integral uses the interaction between criteria and provides the final score for each alternative. Using an illustrative example, we present the applicability of this model in a multi-criteria evaluation problem.

The rest of the paper is organized as follows. In section 2 we present the proposed methodology describing the combination of FLPRAHP, Sugeno $\lambda$-measure and Choquet integral for multi-

\footnotetext{
* Corresponding author
} 
criteria evaluation problems. In section 3 we detail an illustrative example showing the step by step applicability of the proposed method and finally, some conclusions and future directions of the work are included in section 4.

\section{METODOLOGY}

As illustrated in figure 1 the proposed model consists of 2 steps. In the first step the model provides the basis for capturing users preferences which are always uncertain. This step is based on FLPRAHP model. The most challenging issue in this step is the required number of comparisons to determine user's preferences about each criterion. Afterwards, another challenging issue is to measure the interaction between each criterion. In order to cope with this problem, in the second step, we use the determined user's preferences as fuzzy importance of criteria to calculate interaction between them using Sugeno $\lambda$-measure approach. Then Choquet integral is used to aggregate users' preferences and criteria values to calculate final score of each alternative in the fuzzy hierarchical interactive multi-criteria engine.
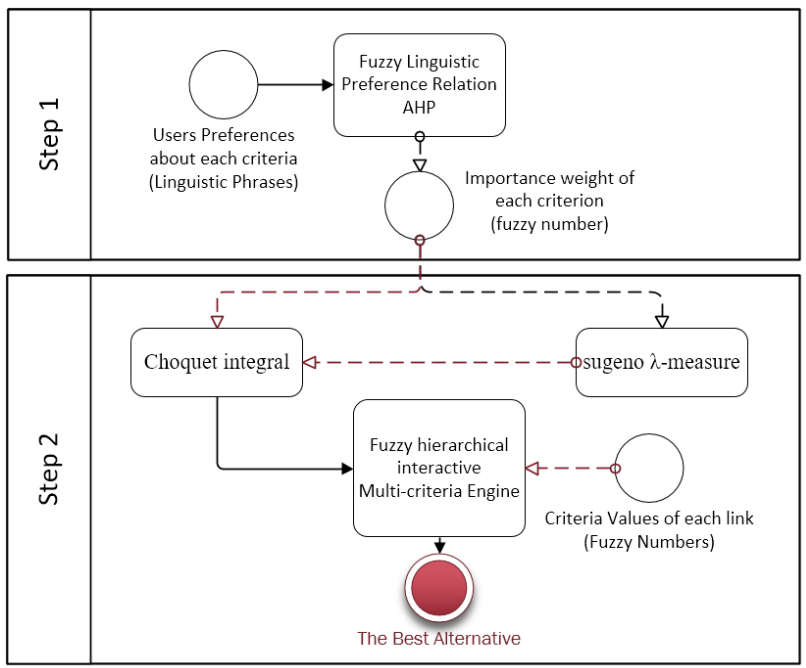

Figure 1. The proposed multi-criteria evaluation model

\subsection{Conventional Fuzzy AHP}

The Analytical Hierarchical Process (AHP) is one of the extensively used multi-criteria decision making methods (Saaty 1980). Although this method is easy to understand and it can model expert opinions through pairwise comparison, however, the conventional AHP cannot process imprecise or vague information (Laarhoven and Pedrycz 1983, Kahraman et.al. 2003, Wang and Chen 2008). In conventional AHP, decision makers compare criteria using crisp judgments. However, in the real situations most experts can just use their judgments regarding criteria relative meaning which are usually vague. It is the essence of the AHP that human judgments, and not just the underlying information, can be used in performing the evaluations. To model the ambiguity in judgments and also uncertainty in criteria values fuzzy extensions of AHP has been introduced. However, the most challenging issue of these methods are to maintain the comparisons consistence. The FLPRAHP provides a method to capture the experts' preferences about criteria using fuzzy linguistic phrases and calculates importance weight of each criterion using least possible number of comparisons while maintains consistency (Wang and Chen 2008). The steps of the conventional Fuzzy AHP are as follows: Step 1: Hierarchical structure construction by placing the goal of the desired problem on the top level of the hierarchical structure, the evaluation criteria on the middle levels and the alternatives on the bottom level.

Step 2: Constructing the fuzzy judgment matrix $\widetilde{A}$. The fuzzy judgment matrix $\widetilde{A}$ in equation 1 is a pairwise comparison of criteria that is constructed by assigning linguistic terms, to the pairwise comparisons by asking which one of two criteria is more important.

$\tilde{A}=\left[\begin{array}{cccc}\tilde{1} & \tilde{a}_{12} & \ldots & \tilde{a}_{1 n} \\ \tilde{a}_{21} & \tilde{1} & \cdots & \tilde{a}_{2 n} \\ \vdots & \vdots & \ddots & \vdots \\ \tilde{a}_{n 1} & \tilde{a}_{n 2} & \cdots & \tilde{1}\end{array}\right]=\left[\begin{array}{cccc}\tilde{1} & \tilde{a}_{12} & & \tilde{a}_{1 n} \\ \tilde{a}_{12}{ }^{-1} & \tilde{1} & & \tilde{a}_{2 n} \\ \vdots & \vdots & \ddots & \vdots \\ \tilde{a}_{1 n}{ }^{-1} & \tilde{a}_{2 n}{ }^{-1} & \cdots & \tilde{1}\end{array}\right]$

where $\tilde{a}_{i j}$ is the fuzzy number from table 1 resulted by comparing $i$ th and $j$ th criteria.

Table 1. Membership function of linguistic scales

\begin{tabular}{l|l}
\hline Fuzzy numbers & Linguistic scales \\
\hline$\widetilde{\mathbf{1}}$ & Equally important \\
\hline$\widetilde{\mathbf{3}}$ & Weakly important \\
\hline$\widetilde{\mathbf{5}}$ & Essentially important \\
\hline$\widetilde{\mathbf{7}}$ & Very strong important \\
\hline$\widetilde{\mathbf{9}}$ & Absolutely important \\
\hline$\widetilde{\mathbf{1}}^{\mathbf{1}} \cdot \widetilde{\mathbf{3}}^{\mathbf{- 1}} \cdot \widetilde{\mathbf{5}}^{\mathbf{- 1}} \cdot \widetilde{\mathbf{7}}^{\mathbf{- 1}} \cdot \widetilde{\mathbf{9}}^{\mathbf{- 1}}$ & Relative less important \\
\hline
\end{tabular}

Step 3: Calculating fuzzy weights of each criterion. The fuzzy weights of each criterion are calculated using equation 2 (Wang and Chen 2008).

$$
\begin{aligned}
& \tilde{r}_{i}=\left[\tilde{a}_{i 1} \otimes \tilde{a}_{i 2} \otimes \ldots \otimes \tilde{a}_{i n}\right]^{\frac{1}{n}} \quad \forall i=1,2, \ldots, n \\
& \widetilde{w}_{i}=\frac{\tilde{r}_{i}}{\tilde{r}_{1} \oplus \ldots \oplus \tilde{r}_{n}}
\end{aligned}
$$

Where $\widetilde{w}_{i}$ is the importance weights of $i$ th criterion.

Step 4: Hierarchical layer sequencing. The final fuzzy weight value of each alternative is calculated by hierarchical layer sequencing using equation 3 .

$$
\widetilde{U}_{i}=\sum_{j=1}^{n} \widetilde{w}_{j} \cdot \tilde{r}_{i j} \quad . \widetilde{U}_{i}=(\text { l.m.u) }
$$

Where $\tilde{r}_{i j}$ is the fuzzy value of the $j$ th criterion, $\widetilde{U}_{i}$ is a fuzzy number shows the final score of $i$ th criterion.

Step 5: Ranking alternatives.

To prepare alternative for ranking at the final step, one approach is defuzzification which transform fuzzy numbers to crisp ones. Equation 4 shows one of the simplest methods named weighted fuzzy mean.

$X\left(\widetilde{U}_{i}\right)=(l+m+u) / 3$

Where $1, \mathrm{~m}$ and $\mathrm{u}$ are lower, mid and upper band of fuzzy number $\widetilde{U}_{i}$, and $X\left(\widetilde{U}_{i}\right)$ is fuzzy mean of $\widetilde{U}_{i}$ which can be used to determine the optimum alternative.

When the number of criteria become large the number of comparisons as well as maintaining them consistent would be a challenging issue. Here we propose to use FLPRAHP methods which solve these problems. Following we explain the FLPRAHP in sections 2.2 .

\subsection{Fuzzy Linguistic Preference Relation AHP}

In the second step of conventional fuzzy AHP described in section 2.1, the amount of comparison can be reduced using the relationship between elements of the matrix $\widetilde{C}[12,13,15]$. Given that the fuzzy positive matrix $\tilde{A}=\left(\tilde{a}_{i j}\right)$ is reciprocal which means that $\tilde{a}_{j i}=\tilde{a}_{i j}{ }^{-1}$ where $\tilde{a}_{i j} \in[1 / 9.9]$, the fuzzy preference relation matrix $\tilde{P}=\left(\tilde{p}_{i j}\right)$ where $\tilde{p}_{i j} \in[0.1]$ can be calculated using transformation in equation 5 (Wang and Chen 2008).

$\tilde{p}_{i j}=\frac{1}{2}\left(1+\log _{9} a_{i j}\right) \cdot \tilde{p}_{i j}=\left(p_{i j}^{L} \cdot p_{i j}^{M} \cdot p_{i j}^{R}\right)$ 
Where $p_{i j}^{L}, p_{i j}^{M}$ and $p_{i j}^{R}$ are left, mid and right band of fuzzy number $\tilde{p}_{i j}$.

For $\tilde{A}=\left(\tilde{a}_{i j}\right)$ being consistent, $\tilde{a}_{i k}$ should be equal to $\tilde{a}_{i j} \otimes \tilde{a}_{j k}$. Taking logarithm on both sides, equation 6 yields (Wang and Chen 2008).

$\tilde{a}_{i j} \otimes \tilde{a}_{j k} \cong \tilde{a}_{i k}$

$\log _{9} a_{i j} \oplus \log _{9} a_{j k}=\log _{9} a_{i k}$

$\log _{9} a_{i j} \oplus \log _{9} a_{j k} \ominus \log _{9} a_{i k}=0$

$\log _{9} a_{i j} \oplus \log _{9} a_{j k} \oplus \log _{9} a_{k i}=0$

$\frac{1}{2}\left(1+\log _{9} a_{i j}\right) \oplus \frac{1}{2}\left(1+\log _{9} a_{j k}\right) \oplus \frac{1}{2}\left(1+\log _{9} a_{k i}\right)=\frac{3}{2}$

Substituting $\frac{1}{2}\left(1+\log _{9} a_{i j}\right)$ from equation 5 , the relationship between elements of matrix $\tilde{A}$ can be determined as detailed in equation 7.

$\tilde{p}_{i j} \oplus \tilde{p}_{j k} \oplus \tilde{p}_{k i}=\frac{3}{2}$

$p_{i j}^{L}+p_{j k}^{L}+p_{k i}^{R}=\frac{3}{2}$

$p_{i j}^{M}+p_{j k}^{M}+p_{k i}^{M}=\frac{3}{2}$

$p_{i j}^{R}+p_{j k}^{R}+p_{k i}^{L}=\frac{3}{2}$

These relationships for more than three criteria are as equation 8 (Wang and Chen 2008).

$p_{i(i+1)}^{L}+p_{(i+1)(i+2)}^{L}+\cdots+p_{(j-1) j}^{L}+p_{j i}^{R}=\frac{(j-i+1)}{2}$

$p_{i(i+1)}^{M}+p_{(i+1)(i+2)}^{M}+\cdots+p_{(j-1) j}^{M}+p_{j i}^{M}=\frac{(j-i+1)}{2}$

$p_{i(i+1)}^{R}+p_{(i+1)(i+2)}^{R}+\cdots+p_{(j-1) j}^{R}+p_{j i}^{L}=\frac{(j-i+1)}{2}$

By using these relationships, the required comparisons for $n$ criteria will be reduced from $n(n-1) / 2$ to just $n-1$ comparisons while the consistency is maintained.

Notably, if the values of the obtained matrix $\tilde{P}$ with elements $\tilde{p}_{i j}$ in the interval $[-c .1+c](c>0)$ are not in the interval $[0,1]$, the obtained fuzzy numbers would need to be transformed via a transformation function to preserve the reciprocity and additive consistency. Equation 9 is used to transform the matrix so that all the entries satisfy this condition (Wang and Chen 2008).

$f:[-c .1+c] \rightarrow[0.1]$

$f\left(P^{L}\right)=\frac{p^{L}+c}{1+2 c}$

$f\left(x p^{M}\right)=\frac{p^{M}+c}{1+2 c}$

$f\left(p^{R}\right)=\frac{p^{R}+c}{1+2 c}$

Where $\mathrm{p}$ is the fuzzy preference relation and $\mathrm{c}$ can be determined using the max and min bound of the $p$ values in the pairwise comparison matrix.

\subsection{Sugeno $\lambda$-Measure}

Sugeno (1974) introduced a specific class of fuzzy measures, which are now known as Sugeno $\lambda$-measure. Let $X=\left\{\mathrm{x}_{1}, \mathrm{x}_{2}, \ldots \mathrm{x}_{\mathrm{n}}\right\}$ be a finite set and $\lambda \in(-1,+\infty)$. A Sugeno $\lambda$-measure is a function $\mathrm{g}: 2^{\mathrm{x}} \rightarrow[0,1]$ which is defined in equation 10 (Sugeno 1974).

1. $\mathrm{g}(\mathrm{X})=1$

2. if $A . B \subseteq X$ where $A . B \in 2^{x}$ and $A \cap B=\emptyset$ then $g(A \cup B)=g(A)+g(B)+\lambda g(A) g(B)$

As a convention, the value of $\mathrm{g}$ at a singleton $\left\{\mathrm{x}_{\mathrm{i}}\right\}$ is called a density and is denoted by $\mathrm{g}_{\mathrm{i}}=\mathrm{g}\left(\left\{\mathrm{x}_{\mathrm{i}}\right\}\right)$. Having $\mathrm{g}_{\mathrm{i}}$ for each singleton, $\lambda$ can be calculated using the equation 11 . $\lambda+1=\prod_{i=1}^{n}\left(1+\lambda g_{i}\right) \quad . \lambda \in(-1 . \infty)$

The $\lambda$ can then be used in equation 10 to calculate the measure for any subsets of $X$ which can be interpreted as the synergy of the criteria in that subset.

Measures from this class are close to the probability measures in the following sense: similarly to the case of probability measures, if we know $g(A)$ and $g(B)$ for two disjoint sets, we can still reconstruct the degree $g(A \cup B)$. The difference is that this reconstructed value is no longer the sum $g(A)+g(B)$. When $\lambda=0$, the Sugeno measure transforms into the additivity probability measure. From this viewpoint, the value $\lambda$ describes how close the given Sugeno measure is to a probability measure.

\subsection{Choquet Integral}

Capacity and Choquet integral, introduced by Choquet (1953), were applied in statistical and potential theory. It was initially used in statistical mechanics and potential theory, but found its way into decision theory in the 1980 s, where it is used as a way of measuring the expected utility of an uncertain event with nonadditive beliefs.

Let $X=\left\{x_{1}, x_{2}, \ldots, x_{n}\right\}$ be a finite set of criteria and $g: 2^{n} \rightarrow[0,1]$ be a fuzzy measure on the subsets of $X$ showing the importance of them and $f: X \rightarrow[0 . \infty]$ represents the value of each criteria for each alternative, then Choquet integral of function $f$ with respect to the fuzzy measure $g$ is defined in equation 12 (Labreuche and Grabisch 2003).

$\int f d g=\sum_{i=1}^{n}\left(f\left(x_{i}\right)-\mathrm{f}\left(x_{i-1}\right)\right) g\left(A_{i}\right)$

Where $A_{i} \subseteq X$ for $i=1.2 .3 \ldots . n$ and $f\left(x_{i}\right)$ is the value of criteria $x_{i}$ which are arranged in the ascending order $f\left(x_{(1)}\right) \leq$ $f\left(x_{(2)}\right) \leq \cdots \leq f\left(x_{(n)}\right)$ where $f\left(x_{(0)}\right)=0$ and $\left\{\mathrm{x}_{(1)}, \mathrm{x}_{(2)}, \ldots\right.$, $\mathrm{X}(\mathrm{n})\}$ are criteria related to each $f$ in the ascending order. Figure 2 illustrates the visual interpretation of the Choquet integral.

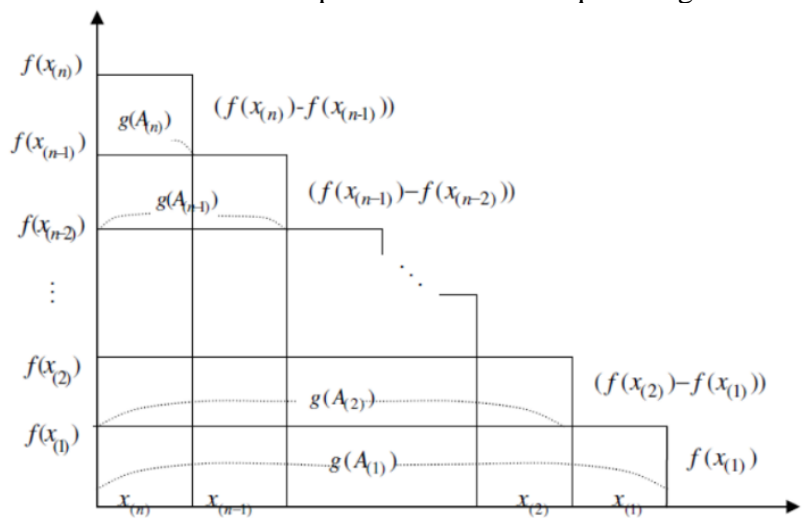

Figure 2. visual illustration of the Choquet integral

As illustrated in this figure the Choquet integral aggregate the criteria values as the area under the chart with non-additive behavior.

\subsection{Fuzzy Hierarchical Interactive Multi-Criteria Engine}

One of the Choquet integral drawbacks is lack of proper structure for the problem. Arranging component of the decision in a hierarchical structure, provides an overall view of the complex relationships between components and helps the decision-maker for better assessment and comparison of alternatives. The proposed fuzzy hierarchical interactive multi-criteria engine is based on the integration of FLPRAHP, Sugeno $\lambda$-measure and 
Choquet integral. Figure 3 illustrates a general typical decision hierarchy. Here we supposed that there are $n$ criteria and $m$ subcriteria which are structured in 2 levels. In the first step FLPRAHP is used to calculate the importance of each criterion. Then in the second step, the weight of interaction between any subsets of criteria are determined using Sugeno $\lambda$-measure. These steps have to be followed for sub-criteria of any criterion. In the third step, Choquet integral is employed in nested to aggregate each alternative values regarding each sub-criteria and then in the upper levels to aggregate calculated scores main criteria. For example, in the 2-level hierarchy illustrated in figure 3 we first use FLPRAHP to determine the weights of each main criterion. Then we use Sugeno $\lambda$-measure to calculate the interaction between these criteria. Afterwards. we again use FLPRAHP to determine the weight of sub-criteria of any main criterion and then use Sugeno $\lambda$-measure again to calculate interaction between them. Then the weights of sub-criteria, their interaction values and the value of alternative $i$ regarding each sub-criterion are aggregated using Choquet integral to calculate the score of alternative i regarding each main criterion. Eventually, these scores, the weights of each main criterion and their interaction values are again aggregated using Choquet integral to calculate the final score of alternative i regarding the goal. These steps have to be followed for each alternative.

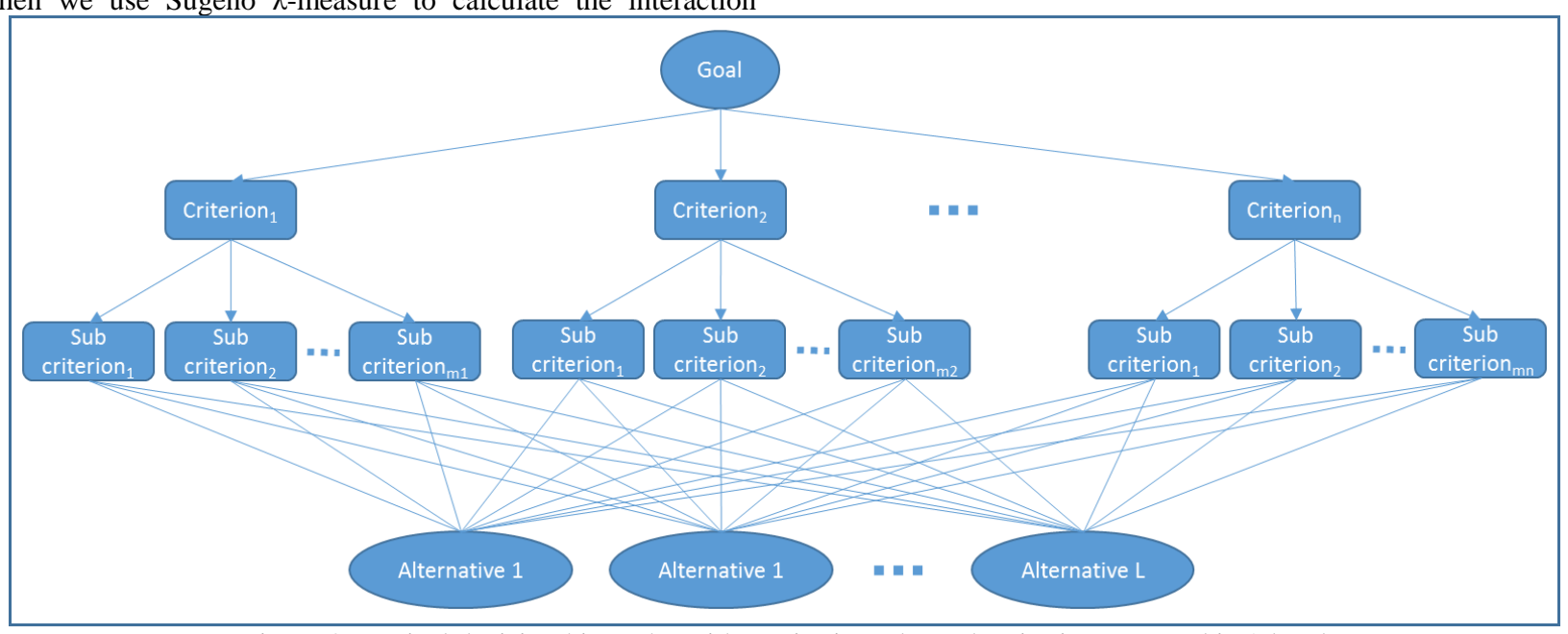

Figure 3. Typical decision hierarchy with $\mathrm{n}$ criteria and $\mathrm{m}$ sub-criteria structured in 2 level

\section{ILLUSTRATIVE EXAMPLE}

The proposed method is demonstrated here using a numerical example. In this example, we are considering determining the best place to establish a new industrial district. We consider four criteria and the aim is to select the optimum location from among various alternatives. The criteria considered in this example are the Potential of the Regional Growth (PRG), Access of District to Cities (ADC), Topography of the District (TOD) and Industrial Infrastructure of District (IID). Regarding the method described in section 2, firstly, we construct the hierarchy as illustrated in figure 3 .

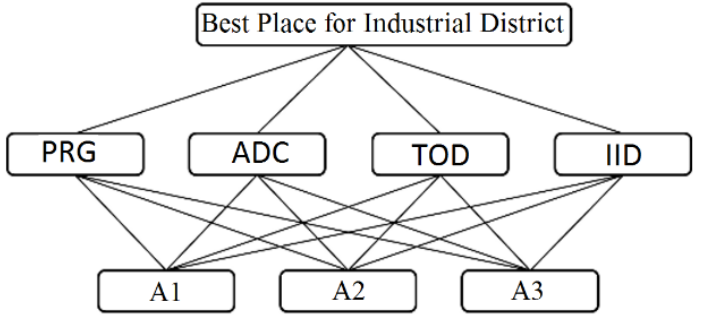

Figure 3. The hierarchy of the problem

$\mathrm{A}_{1}, \mathrm{~A}_{2}$ and $\mathrm{A}_{3}$ are 3 alternatives that are under investigation. Next, we calculate the importance weights of each criterion using FLPRAHP method. Decision makers use the fuzzy linguistic variables in the table 2 to express their opinion while comparing criteria.
Table 2. Fuzzy linguistic assessment variable

\begin{tabular}{ll}
\hline Linguistic variables & Triangular fuzzy number \\
\hline Very poor (VP) & $(0,0,0.1)$ \\
Poor (P) & $(0,0.1,0.3)$ \\
Medium poor (MP) & $(0.1,0.3,0.5)$ \\
Medium (M) & $(0.3,0.5,0.7)$ \\
Medium good (MG) & $(0.5,0.7,0.9)$ \\
Good (G) & $(0.7,0.9,1)$ \\
Very good (VG) & $(0.9,1,1)$ \\
\hline
\end{tabular}

Table 3 shows the pairwise comparison of four criteria. The decision maker provides comparisons just for three criteria pairs of PRG-ADC, ADC-TOD and IID-TOD.

Table 3. Pairwise comparison of four criteria

\begin{tabular}{l|llll}
\hline Goal & PRG & ADC & TOD & IID \\
\hline PRG & $(0.5,0.5,0.5)$ & MG & $?$ & $?$ \\
ADC & $?$ & $(0.5,0.5,0.5)$ & P & $?$ \\
TOD & $?$ & $?$ & $(0.5,0.5,0.5)$ & G \\
IID & $?$ & $?$ & $?$ & $(0.5,0.5,0.5)$
\end{tabular}

Other comparisons are determined using equation 8. Table 4 shows all the determined comparisons.

Table 4. Fuzzy linguistic preference relation matrix of four

\begin{tabular}{l|llll}
\multicolumn{5}{c}{ criteria } \\
\hline Goal & PRG & ADC & TOD & IID \\
\hline PRG & $(0.5,0.5,0.5)$ & $(0.5,0.7,0.9)$ & $(0,0.3,0.7)$ & $(0.2,0.7,1.2)$ \\
ADC & $(0.1,0.3,0.5)$ & $(0.5,0.5,0.5)$ & $(0,0.1,0.3)$ & $(0.2,0.5,0.8)$ \\
TOD & $(0.3,0.7,1)$ & $(0.7,0.9,1)$ & $(0.5,0.5,0.5)$ & $(0.7,0.9,1)$ \\
IID & $(-0.2,0.3,0.8)$ & $(0.2,0.5,0.8)$ & $(0,0.1,0.3)$ & $(0.5,0.5,0.5)$ \\
\hline
\end{tabular}


The matrix in table 4 has entries that are not in the interval $[0,1]$. Thus, they are transformed using equation 9 . Table 5 shows the transformed matrix.

Table 5. Transformed fuzzy linguistic preference relation matrix of four criteria

\begin{tabular}{l|llll}
\hline Goal & PRG & ADC & TOD & IID \\
\hline PRG & $(0.5,0.5,0.5)$ & $(0.5,0.64,0.79)$ & $(0.14,0.36,0.64)$ & $(0.29,0.64,1)$ \\
ADC & $(0.21,0.36,0.5)$ & $(0.5,0.5,0.5)$ & $(0.14,0.21,0.36)$ & $(0.29,0.5,0.71)$ \\
TOD & $(0.36,0.64,0.86)$ & $(0.64,0.79,0.86)$ & $(0.5,0.5,0.5)$ & $(0.64,0.79,0.86)$ \\
IID & $(0,0.36,0.71)$ & $(0.29,0.50,0.71)$ & $(0.14,0.21,0.36)$ & $(0.5,0.5,0.5)$
\end{tabular}

Using this matrix, the importance weight of each criterion is calculated using equation 2 . These weights are expressed in table 6.

Table 6. The importance weight of four criteria

\begin{tabular}{l|ll}
\hline Goal & Average $\left(\tilde{\boldsymbol{r}}_{\boldsymbol{i}}\right)$ & Fuzzy Weight \\
\hline PRG & $(0.36,0.54,0.73)$ & $(0.14,0.27,0.52)$ \\
ADC & $(0.29,0.39,0.52)$ & $(0.11,0.20,0.37)$ \\
TOD & $(0.54,0.68,0.77)$ & $(0.21,0.34,0.54)$ \\
IID & $(0.23,0.39,0.57)$ & $(0.09,0.20,0.41)$ \\
\hline
\end{tabular}

In the next step the score of each alternative regarding each criterion should be determined. Table 7 displays the pairwise comparison matrix for all criteria and alternatives.

Table 7. Pairwise comparison of alternatives regarding each

\begin{tabular}{l|lll}
\multicolumn{4}{c}{ criterion } \\
\hline Goal & A1 & A2 & A3 \\
\hline PRG & & & \\
A1 & $(0.5,0.5,0.5)$ & MP & $?$ \\
A2 & $?$ & $(0.5,0.5,0.5)$ & MP \\
A3 & $?$ & $?$ & $(0.5,0.5,0.5)$ \\
\hline ADC & & & \\
A1 & $(0.5,0.5,0.5)$ & MP & $?$ \\
A2 & $?$ & $(0.5,0.5,0.5)$ & M \\
A3 & $?$ & $?$ & $(0.5,0.5,0.5)$ \\
\hline TOD & & & \\
A1 & $(0.5,0.5,0.5)$ & MP & $?$ \\
A2 & $?$ & $(0.5,0.5,0.5)$ & MG \\
A3 & $?$ & $?$ & $(0.5,0.5,0.5)$ \\
\hline IID & & & \\
A1 & $(0.5,0.5,0.5)$ & M & $?$ \\
A2 & $?$ & $(0.5,0.5,0.5)$ & MP \\
A3 & $?$ & $?$ & $(0.5,0.5,0.5)$ \\
\hline
\end{tabular}

Other comparisons again are determined using equation 8 . Table 8 shows the complete list of comparisons.

Table 8. Fuzzy linguistic preference relation matrix of

\begin{tabular}{l|ccc}
\multicolumn{4}{c}{ alternatives } \\
\hline Goal & A1 & A2 & A3 \\
\hline PRG & & & \\
A1 & $(0.5,0.5,0.5)$ & $(0.1,0.3,0.5)$ & $(-0.3,0.1,0.5)$ \\
A2 & $(0.5,0.7,0.9)$ & $(0.5,0.5,0.5)$ & $(0.1,0.3,0.5)$ \\
A3 & $(0.5,0.9,1.3)$ & $(0.5,0.7,0.9)$ & $(0.5,0.5,0.5)$ \\
\hline ADC & & & \\
A1 & $(0.5,0.5,0.5)$ & $(0.1,0.3,0.5)$ & $(-0.1,0.3,0.7)$ \\
A2 & $(0.5,0.7,0.9)$ & $(0.5,0.5,0.5)$ & $(0.3,0.5,0.7)$ \\
A3 & $(0.3,0.7,1.1)$ & $(0.3,0.5,0.7)$ & $(0.5,0.5,0.5)$ \\
\hline \multicolumn{5}{c}{} & \\
A1 & $(0.5,0.5,0.5)$ & $(0.1,0.3,0.5)$ & $(0.1,0.5,0.9)$ \\
A2 & $(0.5,0.7,0.9)$ & $(0.5,0.5,0.5)$ & $(0.5,0.7,0.9)$ \\
A3 & $(0.1,0.5,0.9)$ & $(0.1,0.3,0.5)$ & $(0.5,0.5,0.5)$ \\
\hline \multicolumn{4}{c}{} \\
A1 & $(0.5,0.5,0.5)$ & $(0.3,0.5,0.7)$ & $(-0.1,0.3,0.7)$ \\
A2 & $(0.3,0.5,0.7)$ & $(0.5,0.5,0.5)$ & $(0.1,0.3,0.5)$ \\
A3 & $(0.3,0.7,1.1)$ & $(0.5,0.7,0.9)$ & $(0.5,0.5,0.5)$ \\
\hline
\end{tabular}

Again, the matrix has entries that are not in the interval $[0,1]$. Thus, they are transformed using equation 9. Table 9 shows the transformed matrix.
Table 9. Transformed fuzzy linguistic preference relations

\begin{tabular}{|c|c|c|c|}
\hline Goal & $\mathrm{A} 1$ & A2 & A3 \\
\hline \multicolumn{4}{|l|}{ PRG } \\
\hline $\mathrm{A} 1$ & $(0.5,0.5,0.5)$ & $(0.25,0.38,0.5)$ & $(0,0.25,0.5)$ \\
\hline A2 & $(0.5,0.63,0.75)$ & $(0.5,0.5,0.5)$ & $(0.25,0.38,0.5)$ \\
\hline A3 & $\begin{array}{l}(0.5,0.75,1) \\
\text { la }\end{array}$ & $(0.5,0.63,0.75)$ & 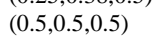 \\
\hline \multicolumn{4}{|c|}{$\mathrm{ADC}$} \\
\hline $\mathrm{Al}$ & $(0.5,0.5,0.5)$ & $(0.17,0.33,0.5)$ & $(0,0.33,0.66)$ \\
\hline $\mathrm{A} 2$ & $(0.5,0.66,0.83)$ & $(0.5,0.5,0.5)$ & $(0.33,0.5,0.66)$ \\
\hline A3 & $(0.33,0.66,1)$ & $(0.33,0.5,0.66)$ & $(0.5,0.5,0.5)$ \\
\hline \multicolumn{4}{|l|}{ TOD } \\
\hline $\mathrm{A} 1$ & $(0.5,0.5,0.5)$ & $(0.1,0.3,0.5)$ & $(0.1,0.5,0.9)$ \\
\hline A2 & $(0.5,0.7,0.9)$ & $(0.5,0.5,0.5)$ & $(0.5,0.7,0.9)$ \\
\hline A3 & $(0.1,0.5,0.9)$ & $(0.1,0.3,0.5)$ & $(0.5,0.5,0.5)$ \\
\hline \multicolumn{4}{|l|}{ IID } \\
\hline $\mathrm{A} 1$ & $(0.5,0.5,0.5)$ & $(0.33,0.5,0.67)$ & $(0,0.33,0.67)$ \\
\hline $\mathrm{A} 2$ & $(0.33,0.5,0.67)$ & $(0.5,0.5,0.5)$ & $(0.17,0.33,0.5)$ \\
\hline A3 & $(0.33,0.67,1)$ & $(0.5,0.67,0.83)$ & $(0.5,0.5,0.5)$ \\
\hline
\end{tabular}

Using this matrix, the score of each alternative regarding each criterion is calculated using equation 2. These scores are expressed in table 10 .

Table 10. Decision table

\begin{tabular}{llcc}
\hline Criteria: weights & A1 & A2 & A3 \\
\hline PRG: $(0.14,0.27,0.52)$ & $(0.14,0.25,0.43)$ & $(0.23,0.33,0.5)$ & $(0.27,0.42,0.64)$ \\
ADC: $(0.11,0.20,0.37)$ & $(0.11,0.26,0.53)$ & $(0.23,0.37,0.64)$ & $(0.20,0.37,0.69)$ \\
TOD: $(0.21,0.34,0.54)$ & $(0.11,0.29,0.66)$ & $(0.25,0.42,0.8)$ & $(0.11,0.29,0.66)$ \\
IID: $(0.09,0.20,0.41)$ & $(0.14,0.29,0.58)$ & $(0.17,0.29,0.53)$ & $(0.23,0.41,0.74)$ \\
\hline
\end{tabular}

So far, we calculate the weight of each criterion and the score of each alternatives regarding to each criterion. In this step we aim to calculate the synergy of the criteria using Sugeno $\lambda$-measure. First we should calculate $\lambda$ using equation 11 .

$$
\begin{aligned}
& \mathrm{g}_{1}=\mathrm{g}(\{\mathrm{PRG}\})=(0.14,0.27,0.52) \\
& \mathrm{g}_{2}=\mathrm{g}(\{A D C\})=(0.11,0.20,0.37) \\
& \mathrm{g}_{3}=\mathrm{g}(\{T O D\})=(0.21,0.34,0.54) \\
& \mathrm{g}_{4}=\mathrm{g}(\{I I D\})=(0.09,0.20,0.41) \\
& \begin{aligned}
1+\lambda=\prod\left(1+\lambda \mathrm{g}_{\mathrm{i}}\right) \rightarrow 1+\lambda \\
=(1+0.31 \lambda) \times(1+0.23 \lambda) \\
\times(1+0.36 \lambda) \times(1+0.24 \lambda) \rightarrow \lambda \\
=0 .-0.31 \stackrel{\lambda \in(-1 . \infty)}{\Longrightarrow} \lambda=-0.31
\end{aligned}
\end{aligned}
$$

According to equation $11 \lambda \in(-1 . \infty)$. For $\lambda=0$, $g$ is an additive measure so we take $\lambda=-0.31$. Table 11 shows the $\lambda$-measures of all subsets of criteria which are calculated using equation 10 .

Table 11. $\lambda$-measures of all subsets of criteria

\begin{tabular}{l|l}
\hline \multicolumn{1}{c|}{ Goal } & \multicolumn{1}{c}{$\lambda$-measures } \\
\hline $\mathbf{g}(\{$ PRG. ADC $\})$ & $(0.25,0.45,0.83)$ \\
$\mathbf{g}(\{$ PRG. TOD $\})$ & $(0.34,0.58,0.97)$ \\
$\mathbf{g}(\{$ PRG. IID $\})$ & $(0.23,0.45,0.86)$ \\
$\mathbf{g}(\{$ ADC. TOD $\})$ & $(0.3,0.52,0.85)$ \\
$\mathbf{g}(\{$ ADC. IID $\})$ & $(0.2,0.39,0.73)$ \\
$\mathbf{g}(\{$ TOD. IID $\})$ & $(0.29,0.52,0.88)$ \\
$\mathbf{g}(\{$ PRG. ADC. TOD $\})$ & $(0.44,0.36,1.59)$ \\
$\mathbf{g}(\{$ PRG. ADC. IID $\})$ & $(0.33,0.63,1.13)$ \\
$\mathbf{g}(\{$ PRG. TOD. IID $\})$ & $(0.42,0.75,1.26)$ \\
$\mathbf{g}(\{$ ADC. TOD. IID $\})$ & $(0.39,0.69,1.15)$ \\
\hline
\end{tabular}

In the final step the final score of each alternative is calculated using Choquet integral with equation 16. Table 12 express the final score of each alternative.

Table $\mathrm{x}$. The final results of the proposed hybrid algorithm

\begin{tabular}{lccc}
\hline Goal & A1 & A2 & A3 \\
\hline Choquet Integral & $(-0.20,0.27,1.57)$ & $(-0.13,0.36,1.59)$ & $(-0.18,0.37,1.92)$ \\
Deffuzification & 0.55 & 0.61 & 0.70 \\
\hline
\end{tabular}


The results showed that Alternative 3 is the place for creating a new industrial district.

\section{CONCLUSION AND FUTURE DIRECTIONS}

This paper proposed a new multi-criteria evaluation model based on the combination of fuzzy AHP method, Choquet integral and Sugeno $\lambda$-measure. In most of the problems in the real world criteria, sub-criteria and alternatives are interdependence and involve uncertainties from different sources. So the proposed model in this paper, employed simplicity and flexibility of AHP and used the fuzzy linguistic preference relation AHP model to model the uncertainties and also utilize Sugeno $\lambda$-measure and Choquet integral to model the interaction between the criteria and sub-criteria and aggregate them to determine the final score of each alternative. finally, a step by step illustrative example shows the efficiency and flexibility of the proposed model.

\section{REFERENCES}

Greco S., Mousseau V. and R. Slowinski (2014). "Robust ordinal regression for value functions handling interacting criteria". European Journal of Operational Research 239(3):711-30.

Grabisch, M. (1996). "The representation of importance and interaction of features by fuzzy measures". Pattern Recognition Letters, 17(6):567-575.

Grabisch, M. (1995). "Fuzzy integral in multicriteria decision making". Fuzzy Sets and Systems, 69(3), 279-298.)

Grabisch M., Murofushi T. and M. Sugeno, (2000). "Fuzzy Measure and Integrals". Physica-Verlag publication, New York.

Kahraman C., Ruan D. and I. Dogan, (2003) "Fuzzy group decision making for facility location selection", Information Sciences 157:135-153

Kojadinovic I., (2002). "Modelling interaction phenomena using fuzzy measures: On the notions of interaction and independence". Fuzzy Sets and Systems, 135(3):317-340.

Laarhoven P.J.M. and W. Pedrycz (1983). "A fuzzy extension of Saaty's priority theory", Fuzzy Sets and Systems 11(13):229-241.

Labreuche, Ch. And M. Grabisch, (2003). "The Choquet integral for the aggregation of interval scales in multicriteria decision making". Fuzzy Sets and Systems, 137(1), 11-26.

Saaty T. L., (2005) "Theory and Applications of the Analytic Network Process: Decision Making with Benefits, Opportunities, Costs, and Risks", RWS publications.

Saaty, T.L., (1980). “The Analytic Hierarchy Process.” McGrawHill, New York.

Saaty T. (1996) "Decision making with dependence and feedback: the analytic network process". Pittsburgh: RWS Publications.

Sugeno M., (1974). "Theory of fuzzy integrals and its applications". Ph.D. thesis, Tokyo Institute of Technology, Tokyo, Japan.

Wang T.C. and Y.H. Chen (2008), "Applying fuzzy linguistic preference relations to the improvement of consistency of fuzzy AHP”, Information Sciences 178(19):3755-3765

Wang T.C. and Y.H. Chen (2005), "A new method on decisionmaking using fuzzy linguistic assessment variables and fuzzy preference relations", In The Proceedings of the 9th World Multi-Conference on Systemics, Cybernetics and Informatics, Orlando, pp. 360-363. 